\title{
THE MINERALOGICAL COMPOSITION OF THRACE ZEOLITIC ROCKS AND THEIR POTENTIAL USE AS FEED ADDITIVES AND NUTRITION SUPPLEMENTS
}

\author{
Filippidis A., Kantiranis N. and Tsirambides A. \\ Aristotle University of Thessaloniki, Faculty of Sciences, School of Geology, Department of \\ Mineralogy-Petrology-Economic Geology, 54124 Thessaloniki, Greece, anestis@geo.auth.gr, \\ kantira@geo.auth.gr,ananias@geo.auth.gr
}

\begin{abstract}
Thirty two (32) locations of zeolitic rocks from Thrace (29 from Evros prefecture and 3 from Rhodope prefecture) were investigated for their mineralogical composition by $X$-Ray Diffraction (XRD) method. According to EU Regulation No 651/2013, clinoptilolite of sedimentary origin (zeolitic tuffs) with $\geq 80 \mathrm{wt} \%$ clinoptilolite, $\leq 20$ $w t \%$ clay minerals, free of fibres and quartz, can be used as feed additive for all animal species. The zeolitic rocks of Thrace on average contain, 23-89 wt\% HEUtype zeolite (clinoptilolite-heulandite), $8-45 \mathrm{wt} \%$ mordenite (ten locations), $24 \mathrm{wt} \%$ analcime (one location), 1-11 wt\% mica, 1-11 wt\% clay minerals, 3-37 wt\% quartz, 2-29 wt\% cristobalite, 3-34 wt\% feldspars and 0-22 wt\% amorphous materials. Only one location contains $89 \mathrm{wt} \%$ HEU-type zeolite (clinoptilolite-heulandite), $2 \mathrm{wt} \%$ clay minerals, is free of fibres, but unfortunately it contains $3 \mathrm{wt} \%$ quartz and $2 \mathrm{wt} \%$ cristobalite. Considering the mineralogical composition, the zeolitic rocks of Thrace do not meet the requirements of the EU Regulation No 651/2013, and thus cannot be used as feed additives for all animal species and consequently as nutrition supplements, since the zeolitic rocks in all locations, contain on average 3-37 wt\% quartz, 2-29 wt\% cristobalite, $8-45 \mathrm{wt} \%$ mordenite (fibrous zeolite) in 10 locations, 24-65 wt\% zeolites (HEU-type \pm mordenite \pm analcime) in 28 locations, $74 \mathrm{wt} \% \mathrm{HEU}$ type zeolite in 2 locations and $77 \mathrm{wt} \%$ HEU-type zeolite in 1 location.
\end{abstract}

Keywords: HEU-type zeolite, fibrous zeolites, mordenite, analcime.

\section{Пврі́ $\eta \psi \eta$}

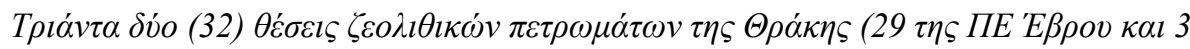

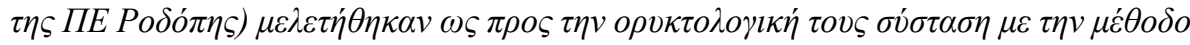

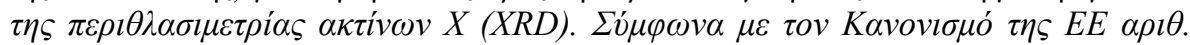

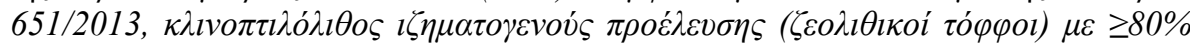

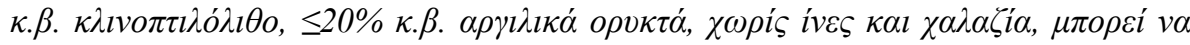

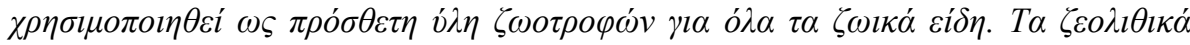

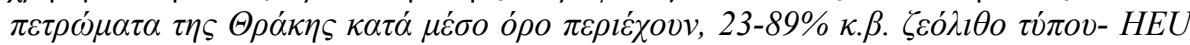

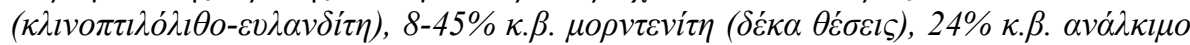

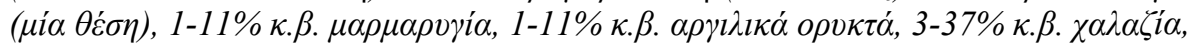

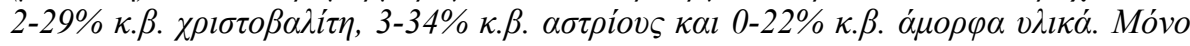

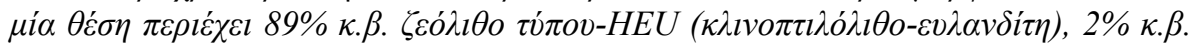

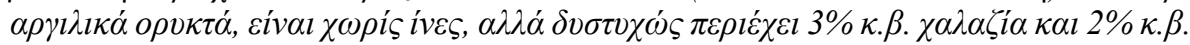

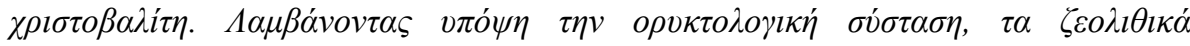




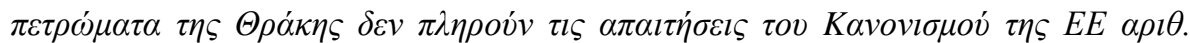

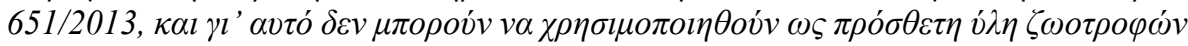

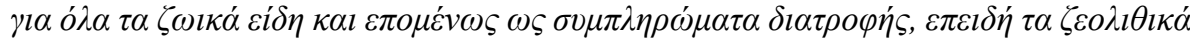

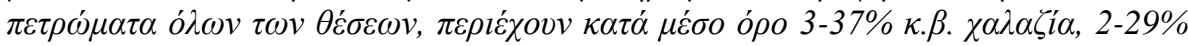

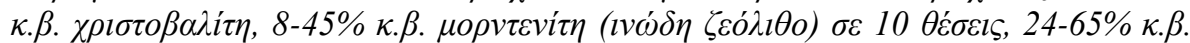

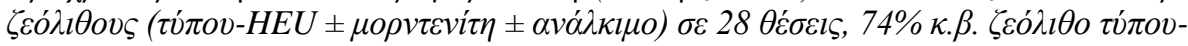

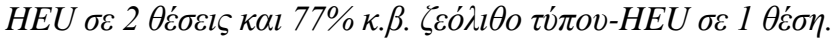

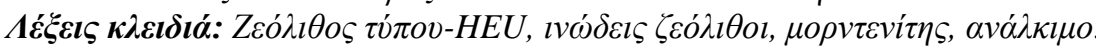

\section{Introduction}

The zeolitic volcaniclastic rock deposit corresponds to a rock which contains high amounts of one or more from the different $(>65)$ phases of zeolites. The zeolite with the numerous applications is the HEU-type zeolite (clinoptilolite-heulandite) that shows tabular crystals and contains micro/nanopores in a framework of channels with 10- and 8-member rings, in dimensions of 7.5x3.1 $\AA, 4.6 \times 3.6$ $\AA$ and 4.7x2.8 $\AA$ (Baerlocher et al. 2007; Mitchell et al., 2012). Only zeolitic tuffs with $\geq 80 \mathrm{wt} \%$ clinoptilolite, $\leq 20 \mathrm{wt} \%$ clay minerals, free of fibres and quartz, can be used as feed additives for all animal species (EU Regulation No 651/2013) and consequently as nutrition supplements. In humans and animals, inhaled or injected or swallowed, fibrous zeolites (mainly erionite and mordenite, and to a lesser extent roggianite and mazzite), as well as the $\mathrm{SiO}_{2}$ minerals (quartz, cristobalite, tridymite), were found to be toxic, carcinogenic and highly pathogenic (Davis, 1993; Driscoll, 1993; Ross et al., 1993).

The zeolitic volcaniclastic rocks of Thrace have been investigated for their mineralogy, petrology and geochemistry by many authors (e.g., Tsirambides et al., 1989, 1993; Kirov et al., 1990; TsolisKatagas and Katagas, 1990; Filippidis, 1993; Koutles et al., 1995; Stamatakis et al., 1996; KassoliFournaraki et al., 2000; Barbieri et al., 2001; Kantiranis et al., 2006a; Filippidis et al., 2007; Marantos et al., 2008; Tzamos et al., 2011). Zeolitic volcaniclastic rocks of Thrace have been tasted in different industrial, environmental and agricultural applications (e.g., Misaelides et al., 1995; Filippidis and Kantiranis, 2007; Filippidis et al., 2008, 2010, 2013, 2015a, b; Filippidis, 2010, 2013; Tsirambides and Filippidis, 2012; Vogiatzis et al., 2012). Some studies concern the use of Thrace zeolitic rocks as feed additives in hens, pigs and lambs (e,g., Tserveni-Gousi et al, 1997; Yannakopoulos et al., 2000; Deligiannis et al., 2005).

The aim of the present study is to investigate the mineralogical composition of the zeolitic rocks from thirty-two (32) locations of Thrace and evaluate their potential use as feed additives (in accordance with the EU Regulation No 651/2013) and as nutrition supplements.

\section{Materials and Methods}

Four (4) to ten (10) representative samples were collected from each zeolitic tuff location of Thrace. The mineralogical composition was determined by the X-Ray Diffraction (XRD) method. The XRD analysis was performed using a Philips PW1710 diffractometer with Ni-filtered $\mathrm{CuK}_{\alpha}$ radiation on randomly oriented powder samples. The counting statistics were: start angle $3^{\circ}$, end angle $63^{\circ}$ and in few samples $43^{\circ}(2 \theta)$, step size $0.02^{\circ}(2 \theta)$, time per step $1 \mathrm{sec}$ and scan speed $0.02^{\circ} / \mathrm{sec}$. Semiquantitative estimates of the abundance of the mineral phases were derived from the XRD data, using the intensity (counts) of certain reflections, the density and the mass absorption coefficient of the identified minerals for $\mathrm{CuK}_{\alpha}$ radiation, the software MAUD-Material Analysis Using Diffraction with the RIETVELD method. The semi-quantitative estimation of the percentage of total amorphous materials was achieved by comparing the area of each broad background hump, which represents the amorphous materials in each sample, with the analogous area of standard mixtures of minerals with different contents of natural amorphous material, scanned under the same conditions (Filippidis and Kantiranis, 2007; Kantiranis et al., 2004, 2005, 2006b). 


\section{Results}

The semi-quantitative mineralogical composition of the zeolitic rock samples are presented in Tables 1-6 and Figures 1 and 2.

In the Petrota area of Evros prefecture, from the 11 locations, the zeolitic rocks contain on average, 47-89 wt \% HEU-type zeolite in 10 locations and $45 \mathrm{wt} \%$ mordenite in 1 location (Table 1, Figure 1). Only in the Ntrista Stream location, the zeolitic rock contains $\geq 80 \mathrm{wt} \%$ HEU-type zeolite (on average $89 \mathrm{wt} \%$ ), $\leq 20 \mathrm{wt} \%$ clay minerals (on average $2 \mathrm{wt} \%$ ), is free of fibres (fibrous zeolites such as mordenite), but unfortunately is not free of quartz $\left(\mathrm{SiO}_{2}\right)$, containing $3 \mathrm{wt} \%$ quartz and $2 \mathrm{wt} \%$ cristobalite $\left(\mathrm{SiO}_{2}\right)$.

Table 1 - Semi-quantitative mineralogical composition (wt\%) of zeolitic rocks from Petrota locations (Evros). HEU-type zeolite: Clinoptilolite-heulandite. Average (range).

\begin{tabular}{|c|c|c|c|c|c|c|c|c|}
\hline $\begin{array}{l}\text { Location } \\
\text { (no. of } \\
\text { samples) }\end{array}$ & $\begin{array}{c}\text { HEU- } \\
\text { type } \\
\text { zeolite }\end{array}$ & $\begin{array}{l}\text { Mord- } \\
\text { enite }\end{array}$ & Mica & $\begin{array}{c}\text { Clay } \\
\text { miner } \\
\text { als }\end{array}$ & Quartz & $\begin{array}{l}\text { Cristo- } \\
\text { balite }\end{array}$ & $\begin{array}{l}\text { Feld- } \\
\text { spars }\end{array}$ & $\begin{array}{l}\text { Amor- } \\
\text { phous }\end{array}$ \\
\hline $\begin{array}{l}\text { Lefki Stream } \\
(4)\end{array}$ & $\begin{array}{c}60 \\
(53-64) \\
\end{array}$ & - & $\begin{array}{c}1 \\
(1-2) \\
\end{array}$ & $\begin{array}{c}2 \\
(1-2) \\
\end{array}$ & $\begin{array}{c}8 \\
(6-9) \\
\end{array}$ & $\begin{array}{c}2 \\
(1-3) \\
\end{array}$ & $\begin{array}{c}27 \\
(24-35) \\
\end{array}$ & - \\
\hline $\begin{array}{l}\text { Tympanistis } \\
\text { (4) }\end{array}$ & $\begin{array}{c}57 \\
(50-62)\end{array}$ & - & $\begin{array}{c}3 \\
(2-4)\end{array}$ & $\begin{array}{c}1 \\
(1-2)\end{array}$ & $\begin{array}{c}9 \\
(7-10)\end{array}$ & $\begin{array}{c}3 \\
(2-4)\end{array}$ & $\begin{array}{c}27 \\
(23-32)\end{array}$ & - \\
\hline Livadakia (4) & $\begin{array}{c}52 \\
(42-60)\end{array}$ & - & $\begin{array}{c}11 \\
(9-12)\end{array}$ & $\begin{array}{c}6 \\
(5-7)\end{array}$ & $\begin{array}{c}11 \\
(8-14)\end{array}$ & $\begin{array}{c}4 \\
(2-6)\end{array}$ & $\begin{array}{c}16 \\
(7-23)\end{array}$ & - \\
\hline $\begin{array}{l}\text { Paliovouni } \\
\text { (4) }\end{array}$ & $\begin{array}{c}52 \\
(39-63) \\
\end{array}$ & - & $\begin{array}{c}2 \\
(1-3) \\
\end{array}$ & $\begin{array}{c}4 \\
(3-5) \\
\end{array}$ & $\begin{array}{c}5 \\
(4-8) \\
\end{array}$ & $\begin{array}{c}4 \\
(2-6) \\
\end{array}$ & $\begin{array}{c}19 \\
(14-27) \\
\end{array}$ & $\begin{array}{c}14 \\
(11-17) \\
\end{array}$ \\
\hline Paliodasos (4) & $\begin{array}{c}60 \\
(57-63) \\
\end{array}$ & - & $\begin{array}{c}1 \\
(1-2) \\
\end{array}$ & $\begin{array}{c}2 \\
(1-3) \\
\end{array}$ & $\begin{array}{c}6 \\
(4-8) \\
\end{array}$ & $\begin{array}{c}2 \\
(1-4) \\
\end{array}$ & $\begin{array}{c}17 \\
(14-20) \\
\end{array}$ & $\begin{array}{c}12 \\
(7-18) \\
\end{array}$ \\
\hline $\begin{array}{l}\text { Fylakio } \\
\text { Omega (4) }\end{array}$ & - & $\begin{array}{c}45 \\
(41-49)\end{array}$ & $\begin{array}{c}2 \\
(1-2) \\
\end{array}$ & $\begin{array}{c}3 \\
(2-4) \\
\end{array}$ & $\begin{array}{c}10 \\
(7-13) \\
\end{array}$ & $\begin{array}{c}15 \\
(13-17)\end{array}$ & $\begin{array}{c}21 \\
(18-24)\end{array}$ & $\begin{array}{c}4 \\
(3-6) \\
\end{array}$ \\
\hline $\begin{array}{l}\text { Mavri Petra } \\
\text { (6) }\end{array}$ & $\begin{array}{c}65 \\
(42-79)\end{array}$ & - & $\begin{array}{c}3 \\
(2-6)\end{array}$ & $\begin{array}{c}2 \\
(1-3)\end{array}$ & $\begin{array}{c}5 \\
(2-6)\end{array}$ & $\begin{array}{c}6 \\
(2-12)\end{array}$ & $\begin{array}{c}16 \\
(8-30)\end{array}$ & $\begin{array}{c}3 \\
(0-9)\end{array}$ \\
\hline Kokkalo (6) & $\begin{array}{c}47 \\
(44-56)\end{array}$ & - & $\begin{array}{c}2 \\
(1-2)\end{array}$ & $\begin{array}{c}3 \\
(2-4)\end{array}$ & $\begin{array}{c}5 \\
(3-6)\end{array}$ & $\begin{array}{c}4 \\
(3-8)\end{array}$ & $\begin{array}{c}17 \\
(14-21)\end{array}$ & $\begin{array}{c}22 \\
(14-27)\end{array}$ \\
\hline Aloni (4) & $\begin{array}{c}59 \\
(55-63) \\
\end{array}$ & - & $\begin{array}{c}2 \\
(1-2) \\
\end{array}$ & $\begin{array}{c}3 \\
(2-4) \\
\end{array}$ & $\begin{array}{c}4 \\
(3-6) \\
\end{array}$ & $\begin{array}{c}4 \\
(2-5) \\
\end{array}$ & $\begin{array}{c}14 \\
(12-19)\end{array}$ & $\begin{array}{c}14 \\
(6-21) \\
\end{array}$ \\
\hline $\begin{array}{l}\text { Ntrista } \\
\text { Stream (6) }\end{array}$ & $\begin{array}{c}89 \\
(83-91) \\
\end{array}$ & - & $\begin{array}{c}1 \\
(1-2) \\
\end{array}$ & $\begin{array}{c}2 \\
(1-3) \\
\end{array}$ & $\begin{array}{c}3 \\
(2-4) \\
\end{array}$ & $\begin{array}{c}2 \\
(1-3) \\
\end{array}$ & $\begin{array}{c}3 \\
(2-7) \\
\end{array}$ & - \\
\hline $\begin{array}{l}\text { Gkazomylos } \\
\text { (10) }\end{array}$ & $\begin{array}{c}60 \\
(47-76)\end{array}$ & - & $\begin{array}{c}2 \\
(1-3)\end{array}$ & $\begin{array}{c}3 \\
(2-11)\end{array}$ & $\begin{array}{c}7 \\
(3-14)\end{array}$ & $\begin{array}{c}7 \\
(2-15)\end{array}$ & $\begin{array}{c}15 \\
(11-21)\end{array}$ & $\begin{array}{c}6 \\
(0-20)\end{array}$ \\
\hline
\end{tabular}




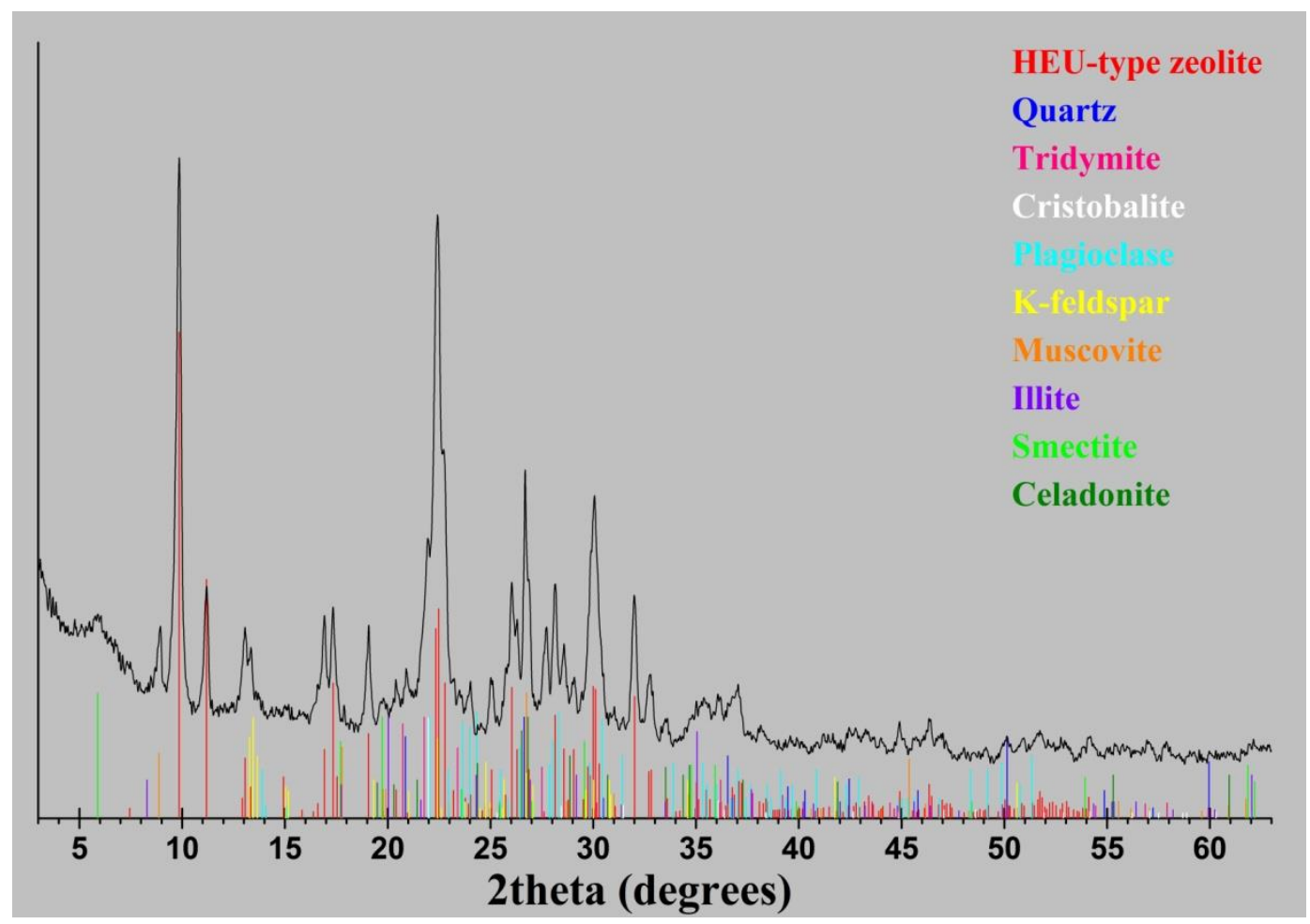

Figure 1 - X-Ray Diffraction pattern of zeolitic rock from Ntrista Stream (Petrota, Evros prefecture).

In the Pentalofos area of Evros prefecture, the zeolitic rocks in all 4 locations contain on average, 55-77 wt $\%$ HEU-type zeolite (clinoptilolite-heulandite), 4-7 wt $\%$ quartz and 3-7 wt $\%$ cristobalite (Table 2).

Table 2 - Semi-quantitative mineralogical composition (wt\%) of zeolitic rocks from Pentalofos locations (Evros). HEU-type zeolite: Clinoptilolite-heulandite. Average (range).

\begin{tabular}{|l|c|c|c|c|c|c|c|}
\hline $\begin{array}{l}\text { Location } \\
\text { (no. of samples) }\end{array}$ & $\begin{array}{c}\text { HEU- } \\
\text { type } \\
\text { zeolite }\end{array}$ & Mica & $\begin{array}{c}\text { Clay } \\
\text { min- } \\
\text { erals }\end{array}$ & Quartz & $\begin{array}{c}\text { Cristo- } \\
\text { balite }\end{array}$ & $\begin{array}{c}\text { Feld- } \\
\text { spars }\end{array}$ & $\begin{array}{c}\text { Amor- } \\
\text { phous }\end{array}$ \\
\hline Palestra (4) & $\begin{array}{c}55 \\
(49-62)\end{array}$ & $\begin{array}{c}3 \\
(2-4)\end{array}$ & $\begin{array}{c}2 \\
(1-3)\end{array}$ & $\begin{array}{c}7 \\
(5-9)\end{array}$ & $\begin{array}{c}7 \\
(4-10)\end{array}$ & $\begin{array}{c}20 \\
(18-27)\end{array}$ & $\begin{array}{c}6 \\
(2-10)\end{array}$ \\
\hline Kapsali Rachi (4) & 74 & 3 & 2 & 5 & 4 & 8 & 4 \\
$(70-77)$ & $(2-4)$ & $(1-3)$ & $(3-7)$ & $(3-6)$ & $(6-10)$ & $(0-6)$ \\
\hline Kyries Toumbes (4) & 74 & 6 & 3 & 4 & 3 & 6 & 4 \\
$(71-77)$ & $(3-10)$ & $(1-5)$ & $(2-6)$ & $(2-6)$ & $(2-10)$ & $(3-5)$ \\
\hline Tympano (6) & $\begin{array}{c}77 \\
(68-86)\end{array}$ & $\begin{array}{c}5 \\
(2-11)\end{array}$ & $\begin{array}{c}5 \\
(2-7)\end{array}$ & $\begin{array}{c}4 \\
(2-5)\end{array}$ & $\begin{array}{c}3 \\
(2-4)\end{array}$ & $\begin{array}{c}6 \\
(3-8)\end{array}$ & - \\
\hline
\end{tabular}

In the Metaxades area of Evros prefecture, the zeolitic rocks in all 3 locations contain on average, 53-55 wt \% HEU-type zeolite (clinoptilolite-heulandite), 7-12 wt \% quartz and 6-21 wt $\%$ cristobalite (Table 3). 
Table 3 - Semi-quantitative mineralogical composition (wt\%) of zeolitic rocks from Metaxades locations (Evros). HEU-type zeolite: Clinoptilolite-heulandite. Average (range).

\begin{tabular}{|l|c|c|c|c|c|c|c|}
\hline $\begin{array}{l}\text { Location } \\
\text { (no. of samples) }\end{array}$ & $\begin{array}{c}\text { HEU-type } \\
\text { zeolite }\end{array}$ & Mica & $\begin{array}{c}\text { Clay } \\
\text { minerals }\end{array}$ & Quartz & $\begin{array}{c}\text { Cristo- } \\
\text { balite }\end{array}$ & $\begin{array}{c}\text { Feld- } \\
\text { spars }\end{array}$ & $\begin{array}{c}\text { Amor- } \\
\text { phous }\end{array}$ \\
\hline Xerovouni (4) & $\begin{array}{c}55 \\
(38-63)\end{array}$ & $\begin{array}{c}7 \\
(4-10)\end{array}$ & $\begin{array}{c}8 \\
(5-11)\end{array}$ & $\begin{array}{c}7 \\
(6-9)\end{array}$ & $\begin{array}{c}6 \\
(3-10)\end{array}$ & $\begin{array}{c}17 \\
(6-41)\end{array}$ & - \\
\hline Gourounorema (5) & $\begin{array}{c}55 \\
(42-69)\end{array}$ & $\begin{array}{c}4 \\
(2-5)\end{array}$ & $\begin{array}{c}6 \\
(2-11)\end{array}$ & $\begin{array}{c}12 \\
(4-22)\end{array}$ & $\begin{array}{c}12 \\
(7-23)\end{array}$ & $\begin{array}{c}11 \\
(4-17)\end{array}$ & - \\
\hline Paliouri (4) & 53 & 2 & 3 & 8 & 21 & 13 & - \\
$(28-67)$ & $(1-3)$ & $(2-5)$ & $(6-12)$ & $(14-35)$ & $(4-22)$ & - \\
\hline
\end{tabular}

In the Dadia-Lefkimmi area of Evros prefecture, the zeolitic rocks in all 3 locations contain on average, 51-53 wt\% HEU-type zeolite (clinoptilolite-heulandite), 6-9 wt\% quartz and 23-29 wt\% cristobalite (Table 4).

Table 4 - Semi-quantitative mineralogical composition (wt\%) of zeolitic rocks from DadiaLefkimmi locations (Evros). HEU-type zeolite: Clinoptilolite-heulandite. Average (range).

\begin{tabular}{|l|c|c|c|c|c|c|c|}
\hline $\begin{array}{l}\text { Location } \\
\text { (no. of samples) }\end{array}$ & $\begin{array}{c}\text { HEU-type } \\
\text { zeolite }\end{array}$ & Mica & $\begin{array}{c}\text { Clay } \\
\text { minerals }\end{array}$ & Quartz & $\begin{array}{c}\text { Cristo- } \\
\text { balite }\end{array}$ & $\begin{array}{c}\text { Feld- } \\
\text { spars }\end{array}$ & $\begin{array}{c}\text { Amor- } \\
\text { phous }\end{array}$ \\
\hline Synoro (4) & 53 & 2 & 3 & 6 & 25 & 11 & - \\
$(46-60)$ & $(1-3)$ & $(2-4)$ & $(3-8)$ & $(19-30)$ & $(6-23)$ & - \\
\hline Xephoto (5) & 51 & 2 & 4 & 7 & 29 & 7 & - \\
$(44-58)$ & $(1-3)$ & $(2-5)$ & $(5-10)$ & $(20-34)$ & $(4-16)$ & - \\
\hline Stavros (4) & 51 & 3 & 4 & 9 & 23 & 10 & - \\
\hline
\end{tabular}

Table 5 - Semi-quantitative mineralogical composition (wt\%) of zeolitic rocks from FeresKirki locations (Evros). HEU-type zeolite: Clinoptilolite-heulandite. Average (range).

\begin{tabular}{|l|c|c|c|c|c|c|c|c|}
\hline $\begin{array}{l}\text { Location } \\
\text { (no. of } \\
\text { samples) }\end{array}$ & $\begin{array}{c}\text { HEU- } \\
\text { type } \\
\text { zeolite }\end{array}$ & $\begin{array}{l}\text { Mord- } \\
\text { enite }\end{array}$ & Mica & $\begin{array}{c}\text { Clay } \\
\text { min- } \\
\text { erals }\end{array}$ & Quartz & $\begin{array}{c}\text { Cristo- } \\
\text { balite }\end{array}$ & $\begin{array}{c}\text { Feld- } \\
\text { spars }\end{array}$ & $\begin{array}{c}\text { Amor- } \\
\text { phous }\end{array}$ \\
\hline $\begin{array}{l}\text { Makrylofos } \\
(7)\end{array}$ & $\begin{array}{c}36 \\
(30-46)\end{array}$ & $\begin{array}{c}13 \\
(11-15)\end{array}$ & $\begin{array}{c}1 \\
(1-3)\end{array}$ & $\begin{array}{c}6 \\
(2-13)\end{array}$ & $\begin{array}{c}21 \\
(14-30)\end{array}$ & $\begin{array}{c}2 \\
(1-4)\end{array}$ & $\begin{array}{c}13 \\
(6-15)\end{array}$ & $\begin{array}{c}8 \\
(2-23)\end{array}$ \\
\hline Kavissos (4) & 38 & 25 & 2 & 9 & 16 & 3 & 3 & 4 \\
$(29-50)$ & $(22-28)$ & $(2)$ & $(7-12)$ & $(5-25)$ & $(1-6)$ & $(0-5)$ & $(0-7)$ \\
\hline Aspra & - & 32 & 2 & 6 & 18 & 2 & 31 & 9 \\
Chomata (7) & $(11-49)$ & $(1-3)$ & $(1-16)$ & $(9-30)$ & $(1-5)$ & $(18-43)$ & $(0-21)$ \\
\hline Laka (4) & 56 & 8 & 2 & 2 & 9 & 3 & 17 & 3 \\
& $(46-66)$ & $(6-10)$ & $(1-3)$ & $(1-4)$ & $(7-13)$ & $(2-5)$ & $(14-22)$ & $(0-5)$ \\
\hline Kapsala (4) & 23 & 21 & 1 & 3 & 19 & 4 & 19 & 10 \\
& $(19-27)$ & $(15-28)$ & $(0-3)$ & $(1-4)$ & $(17-21)$ & $(2-6)$ & $(13-26)$ & $(0-21)$ \\
\hline Nipsa (4) & 33 & 18 & 1 & 8 & 4 & 4 & 29 & 3 \\
& $(30-36)$ & $(15-21)$ & $(0-3)$ & $(6-10)$ & $(2-6)$ & $(2-5)$ & $(26-32)$ & $(2-5)$ \\
\hline $\begin{array}{l}\text { Aetochori } \\
(4)\end{array}$ & 25 & 8 & 1 & 11 & 15 & 4 & 34 & 2 \\
& $(23-28)$ & $(6-10)$ & $(0-2)$ & $(7-13)$ & $(13-17)$ & $(2-6)$ & $(32-37)$ & $(0-4)$ \\
\hline Kirki (4) & 49 & - & 2 & 6 & 10 & 10 & 23 & - \\
\hline
\end{tabular}


In the Feres-Kirki area of Evros prefecture, the zeolitic rocks in all 8 locations contain on average, 23-56 wt\% HEU-type zeolite (clinoptilolite-heulandite) + 8-25 wt\% mordenite in 6 locations, 32 wt\% mordenite in 1 location and 49 wt\% HEU-type zeolite in 1 location (Table 5). The zeolitic rocks of all locations contain on average, 4-21 wt\% quartz and 2-10 wt\% cristobalite (Table 5).

In the Skaloma-Darmeni area of Rhodope prefecture, the zeolitic rocks in all 3 locations contain on average, 23-30 wt\% HEU-type zeolite (clinoptilolite-heulandite) + 20-33 mordenite in 2 locations and $24 \mathrm{wt} \%$ analcime in 1 location (Table 6, Figure 2). The zeolitic rocks of all locations contain on average 10-37 wt\% quartz and 4-16 wt\% cristobalite (Table 6).

Table 6 - Semi-quantitative mineralogical composition (wt\%) of zeolitic rocks from

Skaloma-Darmeni locations (Rhodope prefecture). HEU-type zeolite: Clinoptiloliteheulandite. Average (range).

\begin{tabular}{|c|c|c|c|c|c|c|c|c|c|}
\hline $\begin{array}{l}\text { Location } \\
\text { (no. samples) }\end{array}$ & $\begin{array}{c}\text { HEU- } \\
\text { type } \\
\text { zeolite }\end{array}$ & $\begin{array}{l}\text { Mord- } \\
\text { enite }\end{array}$ & $\begin{array}{l}\text { Anal- } \\
\text { cime }\end{array}$ & Mica & $\begin{array}{l}\text { Clay- } \\
\text { min- } \\
\text { erals } \\
\end{array}$ & $\begin{array}{l}\text { Qua- } \\
\text { rtz }\end{array}$ & $\begin{array}{l}\text { Cristo- } \\
\text { balite }\end{array}$ & $\begin{array}{l}\text { Feld- } \\
\text { spars }\end{array}$ & $\begin{array}{l}\text { Amor- } \\
\text { phous }\end{array}$ \\
\hline $\begin{array}{l}\text { Skaloma: } \\
\text { Avraam (4) }\end{array}$ & $\begin{array}{c}30 \\
(27-33)\end{array}$ & $\begin{array}{c}33 \\
(22-40)\end{array}$ & - & $\begin{array}{c}2 \\
(1-3)\end{array}$ & $\begin{array}{c}6 \\
(2-15) \\
\end{array}$ & $\begin{array}{c}10 \\
(7-16)\end{array}$ & $\begin{array}{c}4 \\
(2-9)\end{array}$ & $\begin{array}{c}9 \\
(4-12)\end{array}$ & $\begin{array}{c}6 \\
(0-11)\end{array}$ \\
\hline $\begin{array}{l}\text { Skaloma: } \\
\text { NE (5) }\end{array}$ & $\begin{array}{c}23 \\
(20-30)\end{array}$ & $\begin{array}{c}20 \\
(17-27)\end{array}$ & - & $\begin{array}{c}1 \\
(0-5)\end{array}$ & $\begin{array}{c}11 \\
(1-15)\end{array}$ & $\begin{array}{c}11 \\
(8-13)\end{array}$ & $\begin{array}{c}16 \\
(9-19)\end{array}$ & $\begin{array}{c}14 \\
(12-15)\end{array}$ & $\begin{array}{c}4 \\
(2-6)\end{array}$ \\
\hline $\begin{array}{l}\text { Darmeni: } \\
\text { Voukefalo (4) }\end{array}$ & - & - & $\begin{array}{c}24 \\
(19-29)\end{array}$ & $\begin{array}{c}1 \\
(0-3)\end{array}$ & $\begin{array}{c}9 \\
(5-20)\end{array}$ & $\begin{array}{c}37 \\
(29-41)\end{array}$ & $\begin{array}{c}5 \\
(2-8)\end{array}$ & $\begin{array}{c}12 \\
(9-14)\end{array}$ & $\begin{array}{c}12 \\
(7-14)\end{array}$ \\
\hline
\end{tabular}

\section{Discussion and Conclusions}

Only clinoptilolite of sedimentary origin (clinoptilolitic zeolitic tuffs) with $\geq 80 \mathrm{wt} \%$ clinoptilolite, $\leq 20 \mathrm{wt} \%$ clay minerals and free of fibres and quartz, can be used (in powder form) as feed additive for all animal species (EU Regulation No 651/2013) and consequently as nutrition supplements. Clinoptilolite of sedimentary origin, belonging to the additive category "technological additives" and to the functional groups "binders" and "anticaking agents", is authorised as an additive in animal nutrition with the conditions laid in the EU Regulation No 651/2013, which is binding in its entirely and directly applicable in all Member States. The EU Regulation No 651/2013, also defines the XRay Diffraction (XRD) as analytical method for the determination of clinoptilolite. In humans and animals, inhaled or injected or swallowed, fibrous zeolites (mainly erionite and mordenite, and to a lesser extent roggianite and mazzite), as well as the $\mathrm{SiO}_{2}$ minerals (quartz, cristobalite, tridymite), were found to be toxic, carcinogenic and highly pathogenic (Davis, 1993; Driscoll, 1993; Ross et al., 1993).

The 32 locations of Thrace (29 in Evros prefecture and 3 in Rhodope prefecture) of zeolitic rocks can be grouped as follows: a) Twenty one locations contain (average values) 47-89 wt\% HEU-type zeolite (clinoptilolite-heulandite), b) Eight locations contain 23-56 wt\% HEU-type zeolite + 8-33 wt $\%$ mordenite, c) Two locations contain $32 \& 45$ wt $\%$ mordenite, d) One location contains $24 \mathrm{wt} \%$ analcime. In all 32 locations the zeolitic rocks contains 3-37 wt $\%$ quartz, 2-29 wt $\%$ cristobalite and $1-11 \mathrm{wt} \%$ clay minerals. 


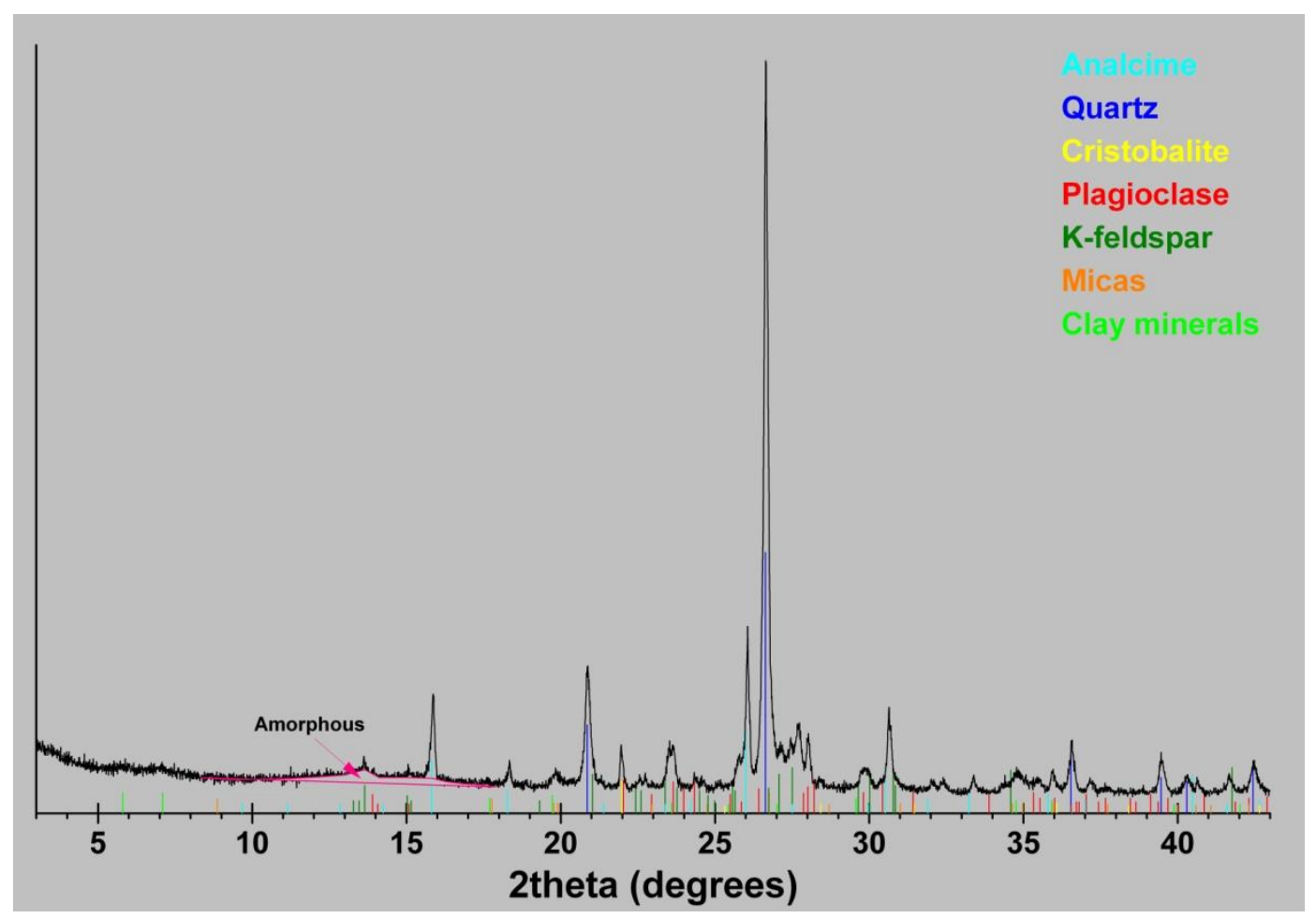

Figure 2 - X-Ray Diffraction pattern of zeolitic rock from Darmeni (Rhodope prefecture).

Only in one location (Ntrista Stream, Petrota, Evros) the zeolitic rock contains $\geq 80 \mathrm{wt} \%$ HEU-type zeolite (clinoptilolite-heulandite) (on average $89 \mathrm{wt} \%$ ), $\leq 20 \mathrm{wt} \%$ clay minerals (on average $2 \mathrm{wt} \%$ ), is free of fibres (fibrous zeolites such as mordenite), but unfortunately contains $3 \mathrm{wt} \%$ quartz and 2 wt $\%$ cristobalite. Concerning the total average zeolite content, one location (Ntrista Stream of Petrota) contains $89 \mathrm{wt} \%$, another one (Tympano of Pentalofos) contains $77 \mathrm{wt} \%$, two (Kapsali Rachi and Kyries Toumpes) contain 74 wt $\%$ and the rest 28 locations of Thrace contain 24-65 wt $\%$ zeolites.

Considering the mineralogical composition described above and presented in Tables 1-6, none of the zeolite rocks of Thrace meet the requirements of the EU Regulation No 651/2013, and thus cannot be used as feed additives for all animal species and consequently as nutrition supplements, since all of them contain on average 3-37 wt\% quartz, 2-29 wt\% cristobalite, 8-45 wt\% mordenite (fibrous zeolite) in 10 locations, 24-65 wt\% zeolites (HEU-type \pm mordenite \pm analcime) in 28 locations, $74 \mathrm{wt} \%$ HEU-type zeolite in 2 locations and $77 \mathrm{wt} \%$ HEU-type zeolite in 1 location.

\section{Acknowledgments}

The authors wish to thank the GEO-VET N. Alexandridis \& Co O.E., for the supply of the zeolitic tuff samples from Ntrista Stream location of Petrota (Evros).

\section{References}

Baerlocher, Ch., McCuster, L.B. and Olson, D.H., 2007. Atlas of Zeolite Framework Types, Amsterdam, Elsevier, 301 pp.

Barbieri, M., Castorina, F., Masi, U., Garbarino, C., Nicoletti, M., Kassoli-Fournaraki, A., Filippidis, A. and Mignardi, S., 2001. Geochemical and isotopic evidence for the origin of rhyolites 
from Petrota (Northern Thrace, Greece) and geodynamic significance, Chemie der Erde, 61, 13-29.

Davis, J.M.G., 1993. In vivo assays to evaluate the pathogenic effects of minerals in rodents. In: Guthrie, G.D.Jr. and Mossman, B.T., eds., Health Effects of Mineral Dusts. Mineralogical Society of America, Washington DC, Reviews in Mineralogy, 28, 471-487.

Deligiannis, K., Lainas, Th., Arsenos, G., Papadopoulos, E., Fortomaris, P., Kufidis, D., Stamataris, C. and Zygoyiannis, D., 2005. The effect of feeding clinoptilolite on food intake and performance of growing lambs infected or not with gastrointestinal nematodes, Livestock Production Science, 96, 195-203.

Driscoll, K.E., 1993. In vitro evaluation of mineral cytotoxicity and inflammatory activity. In: Guthrie, G.D.Jr. and Mossman, B.T., eds., Health Effects of Mineral Dusts, Mineralogical Society of America, Washington DC, Reviews in Mineralogy, 28, 489-511.

EU Regulation No 651/2013. Commission Implementing Regulation (EU) No 651/2013 of 9 July 2013 concerning the authorisation of clinoptilolite of sedimentary origin as a feed additive for all animal species and amending Regulation (EC) No 1810/2005.

Filippidis, A., 1993. New find of moissanite in the Metaxades zeolite-bearing volcaniclastic rocks, Thrace County, Greece, Neues Jahrbuch fur Mineralogie Monatshefte, 11, 521-527.

Filippidis, A., 2010. Environmental, industrial and agricultural applications of Hellenic Natural Zeolite, Hellenic Journal of Geosciences, 45, 91-100.

Filippidis, A., 2013. Industrial and municipal wastewater treatment by zeolitic tuff, Water Today, Jan., V/X, 34-38.

Filippidis, A. and Kantiranis, N., 2007. Experimental neutralization of lake and stream waters from N. Greece using domestic HEU-type rich natural zeolitic material, Desalination, 213, 47-55.

Filippidis, A., Kantiranis, N., Stamatakis, M., Drakoulis, A. and Tzamos, E., 2007. The cation exchange capacity of the Greek zeolitic rocks, Bull. Geol. Soc. Greece, 40(2), 723-735.

Filippidis, A., Apostolidis, N., Paragios, I. and Filippidis, S., 2008. Zeolites clean up, Industrial Minerals, 485, 68-71.

Filippidis, A., Papastergios, G., Apostolidis, N., Filippidis, S., Paragios, I. and Sikalidis, C., 2010. Purification of urban wastewaters by Hellenic Natural Zeolite, Bull. Geol. Soc. Greece, 43(5), 2597-2605.

Filippidis, A., Godelitsas, A., Kantiranis, N., Gamaletsos, P., Tzamos, E. and Filippidis, S., 2013. Neutralization of sludge and purification of wastewater from Sindos industrial area of Thessaloniki (Greece) using natural zeolite, Bull. Geol. Soc. Greece, 47(2), 920-926.

Filippidis, A., Kantiranis, N., Papastergios, G. and Filippidis, S. 2015a. Safe management of municipal wastewater and sludge by fixation of pollutants in very high quality HEU-type zeolitic tuff, J. Basic and Applied Research International, 7(1), 1-8.

Filippidis, A., Papastergios, G., Kantiranis, N. and Filippidis, S., 2015b. Neutralization of dyeing industry wastewater and sludge by fixation of pollutants in very high quality HEU-type zeolitic tuff, J. Global Ecology and Environment, 2(4), 221-226.

Kantiranis, N., Georgakopoulos, A., Filippidis, A. and Drakoulis, A., 2004. Mineralogy and organic matter content of bottom ash samples from Agios Dimitrios power plant, Greece, Bull. Geol. Soc. Greece, 36(1), 320-326.

Kantiranis, N., Filippidis, A. and Georgakopoulos, A., 2005. Investigation of the uptake ability of fly ashes produced after lignite combustion, J. Environmental Management, 76, 119-123.

Kantiranis, N., Chrissafis, C., Filippidis, A. and Paraskevopoulos, K., 2006a. Thermal distinction of HEU-type mineral phases contained in Greek zeolite-rich volcaniclastic tuffs, European J. Mineralogy, 18(4), 509-516.

Kantiranis, N., Filippidis, A., Mouhtaris, T., Paraskevopoulos, K.M., Zorba, T., Squires, C. and Charistos, D., 2006b. EPI-type zeolite synthesis from Greek sulphocalcic fly ashes promoted by $\mathrm{H}_{2} \mathrm{O}_{2}$ solutions, Fuel, $85,360-366$.

Kassoli-Fournaraki, A., Stamatakis, M., Hall, A., Filippidis, A., Michailidis, K., Tsirambides, A. and Koutles, Th., 2000. The Ca-rich clinoptilolite deposit of Pentalofos, Thrace, Greece. In: 
Colella, C. and Mumpton, F.A., eds., Natural Zeolites for the Third Millennium, Napoli, De Frede, 193-202.

Kirov, G.N., Filippidis, A., Tsirambidis, A., Tzvetanov, R.G. and Kassoli-Fournaraki, A., 1990. Zeolite-bearing rocks in Petrota area (Eastern Rhodope Massif, Greece), Geologica Rhodopica, 2, 500-511.

Koutles, Th., Kassoli-Fournaraki, A., Filippidis, A. and Tsirambides, A., 1995. Geology and geochemistry of the Eocene zeolitic-bearing volcaniclastic sediments of Metaxades, Thrace, Greece, Estudios Geologicos, 51, 19-27.

Marantos, I., Markopoulos, Th., Christidis, G.E. and Perdikatsis, V., 2008. Geochemical characteristics of the alteration of volcanic and volcaniclastic rocks in the Feres Basin, Thrace, NE Greece, Clay Minerals, 43, 575-595.

Misaelides, P., Godelitsas, A., Filippidis, A., Charistos, D. and Anousis, I., 1995. Thorium and uranium uptake by natural zeolitic materials, The Science of the Total Environment, 173/174, 237-246.

Mitchell, S., Michels, N.L., Kunze, K. and Perez-Ramirez, J., 2012. Visualization of hierarchically structured zeolite bodies from macro to nano length scales, Nature Chemistry, 4, 825-831.

Ross, M., Nolan, R.P., Langer, A.M. and Cooper, W.C., 1993. Health effects of various mineral dusts other than asbestos. In: Guthrie, G.D.Jr. and Mossman, B.T., eds., Health Effects of Mineral Dusts, Mineralogical Society of America, Washington DC, Reviews in Mineralogy, 28, 361-407.

Stamatakis, M.G., Hall, A. and Hein, J.R., 1996. The zeolite deposits of Greece, Mineralium Deposita, 31, 473-481.

Tserveni-Gousi, A.S., Yannakopoulos, A.L., Katsaounis, N.K., Filippidis, A. and KassoliFournaraki, A., 1997. Some interior egg characteristics as influenced by addition of Greek clinoptilolitic rock material in the hen diet, Archiv fur Geflugelkunde, 61(6), 291-296.

Tsirambides, A. and Filippidis, A., 2012. Exploration key to growing Greek industry, Industrial Minerals, 533, 44-47.

Tsirambides, A., Kassoli-Fournaraki, A., Filippidis, A. and Soldatos, K., 1989. Preliminary results on clinoptilolite-containing volcaniclastic sediments from Metaxades, NE Greece, Bull. Geol. Soc. Greece, 23(2), 451-460.

Tsirambides, A., Filippidis, A. and Kassoli-Fournaraki, A., 1993. Zeolitic alteration of Eocene volcaniclastic sediments at Metaxades, Thrace, Greece, Applied Clay Science, 7, 509-526.

Tsolis-Katagas, P. and Katagas, C., 1990. Zeolitic diagenesis of Oligocene pyroclastic rocks of the Metaxades area, Thrace, Greece, Mineralogical Magazine, 54, 95-103.

Tzamos, E., Kantiranis, N., Papastergios, G., Vogiatzis, D., Filippidis, A. and Sikalidis, C., 2011. Ammonium exchange capacity of the Xerovouni zeolitic tuffs, Avdella area, Evros Prefecture, Greece, Clay Minerals, 46, 179-187.

Vogiatzis, D., Kantiranis, N., Filippidis, A., Tzamos, E. and Sikalidis, C., 2012. Hellenic Natural Zeolite as a replacement of sand in mortar: Mineralogy monitoring and evaluation of its influence on mechanical properties, Geosciences, 2, 298-307.

Yannakopoulos, A., Tserveni-Gousi, A., Kassoli-Fournaraki, A., Tsirambides, A., Michailidis, K., Filippidis, A. and Lutat, U., 2000. Effects of dietary clinoptilolite-rich tuff on the performance of growing-finishing pigs. In: Colella, C. and Mumpton, F.A., eds., Natural Zeolites for the Third Millennium, Napoli, De Frede, 471-481. 